\title{
Optimal Hohmann-Type Impulsive Ellipse-to-Ellipse Coplanar Rendezvous
}

\author{
Xiwen Tian, Yingmin Jia \\ The Seventh Research Division and the Center for Information and Control, School of Automation Science and \\ Electrical Engineering, Beihang University (BUAA), 37 Xueyuan Road, Haidian District \\ Beijing, 100191, China \\ E-mail: tianxiwen123@163.com;ymjia@buaa.edu.cn \\ www.buaa.edu.cn
}

\begin{abstract}
This paper devotes to the problem of ellipse-to-ellipse coplanar rendezvous, where the solution and distribution of Hohmann-type optimal impulsive rendezvous are investigated. The analytical relation between the initial states and rendezvous time are derived for Hohmann-type, and the optimal impulse amplitudes are given thereupon. The distribution boundary of Hohmann-type model is obtained according to the Hohmann transfer and Hohmann with coasts. Simulations are demonstrated to analyze the influences of the solution and distribution.
\end{abstract}

Keywords: Optimal impulsive rendezvous, Hohmann-type rendezvous, ellipse-to-ellipse, optimal distribution.

\section{Introduction}

Optimal impulsive rendezvous is aimed at obtaining minimum-fuel guidance strategy for spacecraft rendezvous, which has attracted considerable attention. Despite that Lawden's necessary conditions ${ }^{1}$ for optimal impulsive trajectories and Lion's improving methods ${ }^{2}$ for non-optimal trajectories have provided some guidelines to solve the optimal problem where the initial states and rendezvous time are specified, the distributions of optimal models cannot be obtained clearly in these way. So far, only Prussing's theory ${ }^{3}$ of optimal impulsive rendezvous on close circular orbits is complete in its theoretical system, which derives the solutions and distributions of optimal impulsive models by solving the primer vector equations and boundary value problem. A reference frame in mean velocity orbit was built by Frank ${ }^{4}$, and showed better performance in describing the impulse locations and magnitudes than the mean radius orbit in Prussing's results. Xie ${ }^{5}$ focused on the selection of reference frame for optimal impulsive rendezvous, and investigated the effect on the classification, distribution and guidance precision. For the case of elliptic orbit rendezvous, Wang ${ }^{6}$ used the state transition matrix given by Yamanaka ${ }^{7}$ to calculate the optimal solution of four-impulse model, but the analytical solution and the distribution are difficult to be achieved. Chen ${ }^{8,9}$ studied the ellipse-to-circle coplanar rendezvous based on his results on the dynamical equations for elliptic orbit rendezvous in low eccentricity, and provided the solutions and distributions of all types optimal models. Motivated by which, our previous work ${ }^{10}$ considered the ellipse-toellipse coplanar rendezvous and obtained the analytical solution and distribution of four-impulse model. In this paper, we will further investigate the Hohmann-type model for optimal impulsive ellipse-to-ellipse coplanar rendezvous.

\section{Dynamics Description}


The relative motion between two spacecrafts in elliptic orbits was derived in our previous work ${ }^{10}$, which is still used in this paper and given as follow:

$$
\left\{\begin{array}{l}
\delta \ddot{r}=3 \delta r+2 \delta \theta \\
\delta \ddot{\theta}=-2 \delta \dot{r}
\end{array}\right.
$$

The initial and terminal states of system (1) are

$$
\begin{aligned}
& x_{0}=\left[x_{01}, x_{02}, x_{03}, x_{04}\right]^{T} \\
& x_{F}=[0,0,0,0]^{T}
\end{aligned}
$$

where

$$
\begin{aligned}
& x_{01}=k_{c}\left(1+e_{c} \cos f_{c}\right)^{-1}-k_{t}\left(1+e_{t} \cos f_{t}\right)^{-1} \\
& x_{02}=\beta \\
& x_{03}=k_{c}^{-\frac{1}{2}} e_{c} \sin f_{c}-k_{t}^{-\frac{1}{2}} e_{t} \sin f_{t} \\
& x_{04}=k_{c}^{-\frac{3}{2}}\left(1+e_{c} \cos f_{c}\right)^{2}-k_{t}^{-\frac{3}{2}}\left(1+e_{t} \cos f_{t}\right)^{2}
\end{aligned}
$$

$\beta$ is the difference of phase angle between two spacecrafts; $e_{c}, e_{t}$ and $f_{c}, f_{t}$ are their eccentricities and true anomalies, respectively.

The states at phase angle $\tau$ was also deduced[10]:

$$
x(\tau)=\left[\begin{array}{c}
2 d_{4}-d_{3} \cos (\tau+\varphi) \\
\beta-3 d_{4} \tau+2 d_{3} \sin (\tau+\varphi)-2 d_{3} \sin \varphi \\
d_{3} \sin (\tau+\varphi) \\
-3 d_{4}+2 d_{3} \cos (\tau+\varphi)
\end{array}\right]
$$

where

$$
\begin{aligned}
& d_{1}=x_{03}, d_{2}=3 x_{01}+2 x_{04} \\
& d_{3}=\sqrt{d_{1}^{2}+d_{2}^{2}}, d_{4}=2 x_{01}+x_{04}, \varphi=\arcsin \left(\frac{d_{1}}{d_{3}}\right)
\end{aligned}
$$

\section{Optimal Hohmann-Type Rendezvous}

The solution to primer vector equations corresponding to system (1) can be given in the following form:

$$
\begin{aligned}
& \lambda_{1}=A \cos \tau+B \sin \tau+2 C \\
& \lambda_{2}=2 B \cos \tau-2 A \sin \tau-3 C \tau+D
\end{aligned}
$$

Hohmann-type model is a special case of optimal twoimpulse rendezvous, where the coefficients of (6) are

$$
A=B=C=0, D= \pm 1
$$

then $\lambda_{1}=0,\left|\lambda_{2}\right|=1$. It can be verified that the necessary conditions of optimal impulsive rendezvous are satisfied for any phase angle $\tau$.

\subsection{Solution of Hohmann transfer}

The impulse direction can be obtained from the solution (6), while the impulse time and magnitudes needed be calculated according to the following boundary value problem:

$$
\left[\begin{array}{ll}
2\left(1-C_{\tau}\right) & 0 \\
4 S_{\tau}-3 \tau_{F} & 0 \\
2 S_{\tau} & 0 \\
-3+4 C_{\tau} & 1
\end{array}\right]\left[\begin{array}{l}
\Delta V_{1} \\
\Delta V_{2}
\end{array}\right]=x\left(\tau_{F}\right)
$$

where $S_{\tau}=\sin \tau, C_{\tau}=\cos \tau, \Delta V_{1}$ and $\Delta V_{2}$ are the magnitudes of two impulse, and $\tau_{F}$ is the rendezvous time. From (4) and (8), we have

$$
\begin{aligned}
& \Delta V_{1}=-\frac{d_{3} \sin \left(\tau_{F}+\varphi\right)}{2 S_{\tau}} \\
& \Delta V_{1}=\frac{-2 d_{4}+d_{3} \cos \left(\tau_{F}+\varphi\right)}{2\left(1-C_{\tau}\right)}
\end{aligned}
$$

then

$$
d_{3}\left(\sin \left(\tau_{F}+\varphi\right)-\sin \varphi\right)=2 d_{4} \sin \tau_{F}
$$

Substituting (5) into (10), it can be obtained that

$$
\sqrt{x_{01}^{2}+d_{1}^{2}} \sin \left(\tau_{F}+\theta\right)=-d_{1}
$$

where

$$
\cos \theta=\frac{x_{01}}{\sqrt{x_{01}^{2}+d_{1}^{2}}}, \sin \theta=-\frac{d_{1}}{\sqrt{x_{01}^{2}+d_{1}^{2}}}
$$

On the other hand, from the second row of (8), it has

$$
\Delta V_{1}=\frac{-\beta+3 d_{4} \tau-2 d_{3} \sin \left(\tau_{F}+\varphi\right)+2 d_{3} \sin \varphi}{\left(4 S_{\tau}-3 \tau_{F}\right)}
$$

Combining (5), (9) and (13), it has

$$
-\frac{3 \tau_{F}\left(x_{01} C_{\tau}+d_{1} S_{\tau}\right)}{2\left(1-C_{\tau}\right)}+2 d_{1}=f_{c}-f_{t}
$$

The appropriate initial states and rendezvous time which satisfy the necessary conditions of Hohmann transfer can be obtained by solving (11) and (14) together, and then, the second impulse $\Delta V_{2}$ can be obtained as

$$
\Delta V_{2}=-d_{4}+\frac{d_{3} \sin \left(\tau_{F}+\varphi\right)}{2 S_{\tau}}
$$

\subsection{Distribution of Hohmann-type model}

The distribution of optimal Hohmann-type rendezvous is to illustrate the existence of feasible solution. To investigate the distribution, rendezvous time is chosen as the $\mathrm{X}$-coordinate and the special phase angle defined below as Y-coordinate: 


$$
\delta \theta_{F}=-\beta+1.5 d_{4} \tau_{F}-d_{3} \sin \left(\tau_{F}+\varphi\right)+d_{3} \sin \varphi(16)
$$

Let $\tau_{\mathrm{Fh}}$ be rendezvous time solved by (11) and (14), and $\delta \theta_{\mathrm{Fh}}$ is the corresponding special phase angle. If $\tau_{F}=\tau_{F h}$ and $\delta \theta_{F}=\delta \theta_{F h}$, then it is just the Hohmann transfer. The two impulses are implemented at $\tau_{1}=0$ and $\tau_{2}=\tau_{F}$. However, when the real rendezvous time is longer than $\tau_{F h}$, the coasts are needed to save the fuel.

If $\tau_{\mathrm{F}}>\tau_{\mathrm{Fh}}$ and $\delta \theta_{\mathrm{F}}=\delta \theta_{\mathrm{Fh}}$, it is a Hohmann model with terminal coast. The two impulses are implemented at $\tau_{1}=0$ and $\tau_{2}=\tau_{\mathrm{Fh}}$, and the residual time $\tau_{\mathrm{F}}-\tau_{\mathrm{Fh}}$ is for terminal coast. The special phase angle $\delta \theta_{\mathrm{F}}$ and rendezvous time $\tau_{\mathrm{F}}$ should satisfy the following relation

$$
\begin{aligned}
\delta \theta_{F}= & \delta \theta_{F h}+1.5 d_{4}\left(\tau_{F}-\tau_{F h}\right) \\
& +d_{3} \sin \left(\tau_{F h}+\varphi\right)-d_{3} \sin \left(\tau_{F}+\varphi\right) \\
\tau_{F h}= & \tau_{2}-\tau_{1}, \tau_{F} \in\left[\tau_{F h},+\infty\right)
\end{aligned}
$$

If $\tau_{F}>\tau_{F h}$ and the special phase angle $\delta \theta_{F}$ satisfies

$$
\begin{aligned}
\delta \theta_{F}= & \delta \theta_{F h}-1.5 d_{4}\left(\tau_{F}-\tau_{F h}\right) \\
& +d_{3} \sin \left(\tau_{F}-\tau_{F h}+\varphi\right)-d_{3} \sin \varphi \\
\tau_{F h}= & \tau_{2}-\tau_{1}, \tau_{F} \in\left[\tau_{F h},+\infty\right)
\end{aligned}
$$

then, after the initial coast for time $\tau_{F}-\tau_{F h}$, the special phase angle will become exactly $\delta \theta_{F h}$. This case is a Hohmann model with initial coast, and the impulses are implemented at $\tau_{1}=\tau_{F}-\tau_{F h}$ and $\tau_{2}=\tau_{F}$.

Let

$$
\begin{aligned}
\delta \theta_{F h 1}= & \delta \theta_{F h}+1.5 d_{4}\left(\tau_{F}-\tau_{F h}\right) \\
& +d_{3} \sin \left(\tau_{F h}+\varphi\right)-d_{3} \sin \left(\tau_{F}+\varphi\right) \\
\delta \theta_{F h 2}= & \delta \theta_{F h}-1.5 d_{4}\left(\tau_{F}-\tau_{F h}\right) \\
& +d_{3} \sin \left(\tau_{F}-\tau_{F h}+\varphi\right)-d_{3} \sin \varphi
\end{aligned}
$$

If $\tau_{F}>\tau_{F h}$ and $\delta \theta_{F h 1}<\delta \theta_{F}<\delta \theta_{F h 2}$, then there exists a Hohmann model with both initial coast and terminal coast. As shown in Fig.1., this case is illustrated in the middle of the curves expressed by (17) and (18), that is the shadow part. Denote $\left(\tau_{F 0}, \delta \theta_{F 0}\right)$ as the intersection point of the curves determined by (16) and (18), then the two impulses are implemented at $\tau_{1}=\tau_{F 0}-\tau_{F h}$ and $\tau_{2}=\tau_{F 0}$. The initial and terminal coast last for time $\tau_{F 0}-\tau_{F h}$ and $\tau_{F}-\tau_{F 0}$, respectively.

From the above, the optimal Hohmann-type impulsive rendezvous has four models, all of whose impulse magnitudes are determined by (9) and (15), and impulse direction is along the tangential direction.

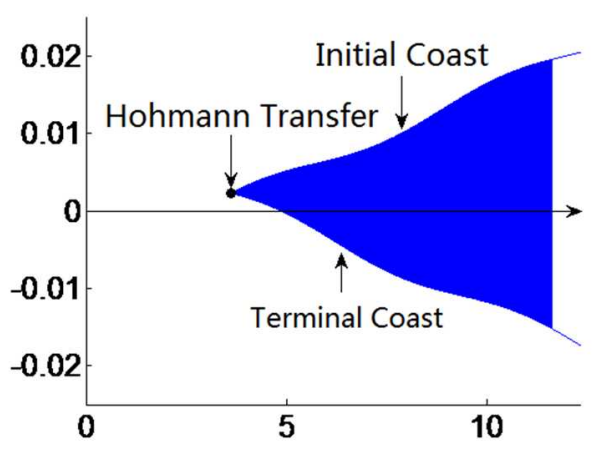

Fig 1 Distribution of Hohmann-type model

\section{Simulations}

In this section, simulation examples are presented to show the guidance performance and distribution of Hohamman-type impulsive rendezvous.

\subsection{Hohmann ellipse-to-ellipse rendezvous}

It is assumed the semi-major axis and eccentricities of the target orbit and chaser orbit are initially $a_{t}=$ $6730(\mathrm{~km}), a_{c}=6750(\mathrm{~km}), e_{t}=0.0005$ and $e_{c}=$ 0.0004 , respectively. Let $\tau_{F}$ and $\beta$ (rad) be the appropriate rendezvous time and initial difference of phase angle, respectively, which satisfy (11) and (14). And denote $R_{r}(\mathrm{~m})$ as the optimal radius of reference frame, $R(\mathrm{~m})$ as the initial relative distance, $\Delta a(\mathrm{~m}), \Delta e$ and $\Delta \theta(\mathrm{rad})$ as the guidance errors.

Table 1. Results of Hohmann impulsive rendezvous

\begin{tabular}{|c|c|c|c|c|}
\hline & 1 & 2 & 3 & 4 \\
\hline $\mathrm{f}_{\mathrm{c}}$ & $30^{\circ}$ & $150^{\circ}$ & $210^{\circ}$ & $330^{\circ}$ \\
\hline$\tau_{\mathrm{F}}$ & 3.62 & 3.44 & 2.80 & 2.68 \\
\hline$\beta$ & $7.12 \mathrm{e}-03$ & $9.68 \mathrm{e}-03$ & $6.04 \mathrm{e}-03$ & $3.46 \mathrm{e}-03$ \\
\hline $\mathrm{R}_{\mathrm{r}}$ & $6.70 \mathrm{e}+06$ & $7.92 \mathrm{e}+06$ & $7.81 \mathrm{e}+06$ & $6.71 \mathrm{e}+06$ \\
\hline$R$ & $4.99 \mathrm{e}+04$ & $7.05 \mathrm{e}+04$ & $4.83 \mathrm{e}+04$ & $2.71 \mathrm{e}+04$ \\
\hline$\Delta \mathrm{a}$ & $6.80 \mathrm{e}+01$ & $3.38 \mathrm{e}+04$ & $3.09 \mathrm{e}+04$ & $2.49 \mathrm{e}+01$ \\
\hline$\Delta \mathrm{e}$ & $3.38 \mathrm{e}-05$ & $4.51 \mathrm{e}-03$ & $4.22 \mathrm{e}-03$ & $8.92 \mathrm{e}-06$ \\
\hline$\Delta \theta$ & $5.82 \mathrm{e}-06$ & $2.87 \mathrm{e}-04$ & $3.50 \mathrm{e}-05$ & $3.80 \mathrm{e}-06$ \\
\hline$\Delta \mathrm{R}$ & $1.29 \mathrm{e}+02$ & $5.14 \mathrm{e}+03$ & $5.71 \mathrm{e}+02$ & $5.08 \mathrm{e}+01$ \\
\hline
\end{tabular}

Simulation results of Hohmann transfer for ellipseto-ellipse rendezvous are demonstrated in Table 1, which shows that: (1) with different true anomalies, even if the other initial states are the same, the 
rendezvous time and initial difference of phase angle which satisfy (11) and (14) varies much; (2) the optimal radius of reference frame also changes with the true anomaly; (3) the guidance precision is high when the chaser initially stays around the perigee.

\subsection{Distribution of Hohmann-type model}

To investigate the distribution of Hohmann-type ellipseto-ellipse rendezvous, we take rendezvous time $\tau_{F}$ as the $\mathrm{X}$-coordinate and $\delta \theta_{F} / d_{4}$ as Y-coordinate. Fig.2 shows the distribution of Hohmann-type model with different true anomalies and eccentricities.

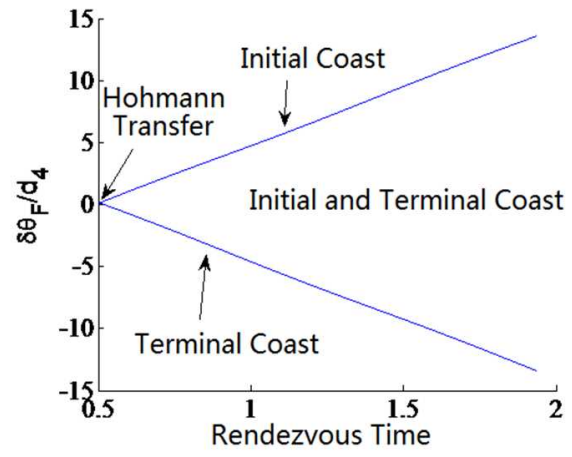

(a) $\left[e_{t}, e_{c}\right]=[0.0004,0.0005], f_{c}=30^{\circ}$

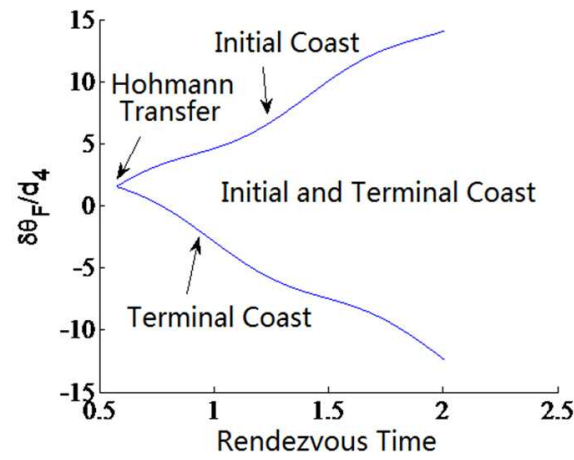

(b) $\left[e_{t}, e_{c}\right]=[0.004,0.005], f_{c}=30^{\circ}$

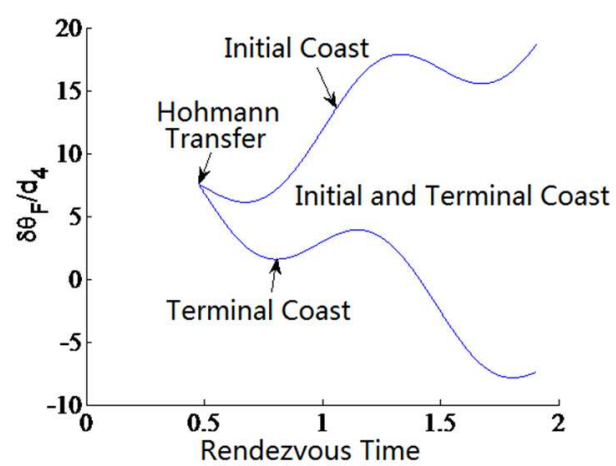

(c) $\left[e_{t}, e_{c}\right]=[0.051,0.05], f_{c}=30^{\circ}$

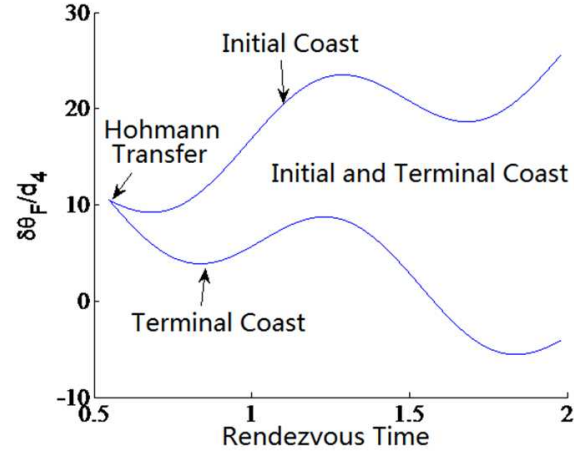

(d) $\left[e_{t}, e_{c}\right]=[0.004,0.005], f_{c}=150^{\circ}$

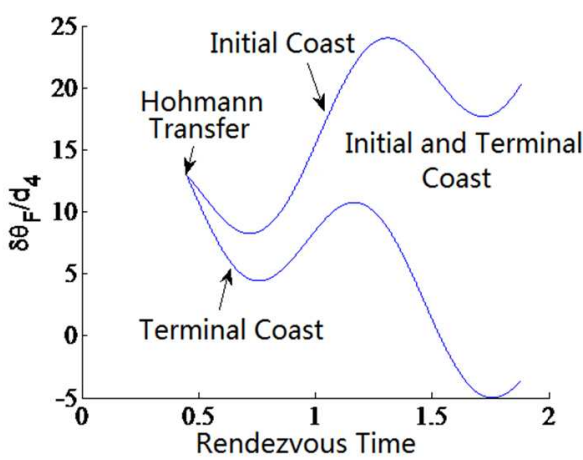

(e) $\left[e_{t}, e_{c}\right]=[0.004,0.005], f_{c}=210^{\circ}$

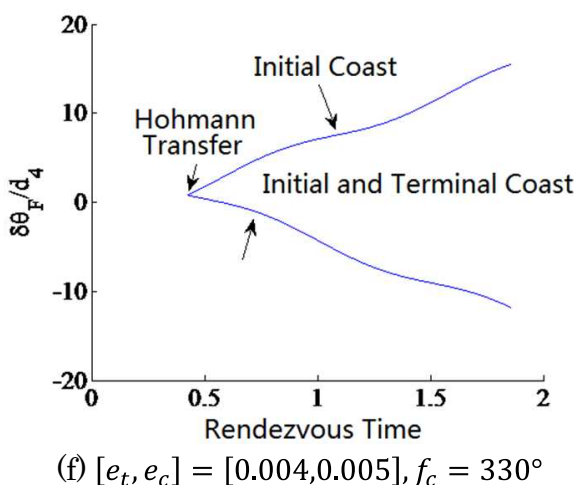

Fig 2 Distributions with different true anomalies and eccentricities

\section{Conclusion}

This paper extends our previous work $^{10}$ to the Hohmann-type optimal impulsive rendezvous. By defining the special phase angle, we derived the analytical solution for Hohmann transfer, and obtained that the optimal Hohmann-type impulsive rendezvous has four models, i.e. Hohmann transfer, Hohmann with initial coast, Hohmann with terminal coast and Hohmann with both coasts. In further research, we will integrate all optimal models in one map, including four- 
impulse, three-impulse, three-impulse with coasts, twoimpulse, two-impulse with coasts, and Hohmann-type.

\section{Acknowledgements}

This work was supported by NSFC (61327807, 61521091, 61520106010, 61134005) and the National Basic Research Program of China (973 Program: 2012CB821200, 2012CB821201)

\section{References}

1. D. F. Lawden, Optimal Trajectories for Space Naviation (London: Butterworhs, 1963).

2. P.M. Lion and M. Handelsman, Primer vector on fixedtime impulsive trajectories, AIAA J., 6(1) (1968) 127-132.

3. J. E. Prussing, Optimal Multi-Impulse Orbital rendezvous, Sc.D. Thesis. (Cambridge: Dept. of Aeronautics and Astronautics, Massachusetts Institute of Technology, 1967).
4. C. L. Frank and L. D. Plexico, Improved solution of optimal impulsive fixed-time rendezvous. J. Spacecraft and Rockets, 19(6) (1982) 521-528

5. Y. C. Xie, C. Q. Chen, H. Zhang and J. Hu, Selection of reference systems for optimal impulsive rendezvous between two near circular orbits (in Chinese), Chinese Space Sci. Technol., 28(2) (2008) 8-14.

6. J. X. Wang, H. X. Baoyin, J. F. Li and F. C. Sun, Optimal four-impulse rendezvous between coplanar elliptical orbits. Phys. Mech. Astron., 54(4) (2011) 792-802.

7. K. Yamanaka and F. Ankersen, New state transition matrix for relative motion on an arbitrary elliptical orbit, J. Guid. Contr. Dynam., 25(1) (2002) 60-66.

8. C. Q. Chen and Y. C. Xie, Dynamical equations for a kind of elliptic orbit rendezvous (in Chinese), Aerospace Contr., 26(5) (2008) 12-17.

9. C. Q. Chen and Y. C. Xie, Optimal impulsive ellipse-tocircle coplanar rendezvous, Sci. in China Series E: Tech. Sci., 52(5) (2009) 1435-1445.

10. X. W. Tian, Y. M. Jia and C. Q. Chen, Optimal fourimpulse ellipse-to-ellipse coplanar rendezvous, Contr. Conf. IEEE, (2016) 5498-5503. 